\title{
LA ESCUELA DE PADRES COMO ESTRATEGIA PEDAGÓGICA PARA FORTALECER EL RENDIMIENTO ACADÉMICO DE LOS ESTUDIANTES ${ }^{44}$
}

[The Escuela de Padres as a strategy to improve the academic performance of students]

Anabella García Torres*

García, A. (2016). La escuela de padres como estrategia pedagógica para fortalecer el rendimiento académico de los estudiantes. Revista PACA, 8, 143-156

\section{Resumen}

El rendimiento académico de los estudiantes es uno de los problemas, que a través de los tiempos y en todos los niveles de la educación, ha ocupado la atención de quienes, de una u otra forma, están comprometidos en la difícil tarea de educar; es por lo anterior, que se llevó a cabo el presente trabajo de investigación, cuyo objetivo se centró en determinar el grado de influencia de la Escuela de Padres como estrategia pedagógica para mejorar el rendimiento académico de los estudiantes de educación básica primaria de la Sede Los Pinos de la Institución Educativa María Cristina Arango. Desde esta perspectiva se llevó a cabo una investigación de estudio

\footnotetext{
${ }^{44}$ Proyecto de investigación: Escuela de padres como estrategia pedagógica para fortalecer el rendimiento académico de los estudiantes de básica primaria en la sede Los Pinos de la Institución Educativa "María Cristina Arango de Pastrana"; desarrollado durante los años 2014 - 2015.

* Magister en Educación, Especialista en Educación Sexual, Especialista en Desarrollo Humano. Orientadora Escolar de la Institución Educativa María Cristina Arango. Email: Ilabeana14@hotmail.com
} 
de casos (carácter comparativo) mediante un enfoque cualitativo, la cual permitió evidenciar la ausencia de compromiso de los padres de familia durante el proceso de formación de sus hijos, lo cual genera como consecuencia un bajo rendimiento académico por parte de los mismos; situación que permitió señalar la importancia de fortalecer la escuela de padres como estrategia educativa. Partiendo de los resultados encontrados se plantea una propuesta pedagógica, la cual ofrece herramientas fundamentales que permite capacitar a la comunidad educativa sobre las acciones oportunas que dan lugar a superar problemáticas propias de la interacción entre padres e hijos, en lo relacionado con la parte afectivaacadémica, hábitos y los que se generen propios de la dinámica del contexto institucional y de los hogares de los estudiantes.

Palabras clave: Estrategia pedagógica, escuela de padres, rendimiento académico.

\section{Abstract}

The academic performance of the students is one of the problems that has become in the main idea of those who are dedicated to this difficult labor of teaching. That is the reason why we carry out this research project, which the principal goal is based on determinate the influence of the "Escuela de Padres" as a strategy to improve the academic performance of Maria Cristina Arango school sede "Los Pinos" students in primary level. We based the research in the different cases (taking into account the qualitative approach), where was proved the lack of commitment from parents to get involve in their children educative process and as a consequence we get children with a low academic performance. This fact let us point out the importance to reinforce the Escuela de Padres as an educative strategy. Based on the results founded, we propose a pedagogical strategy which offers fundamental tools that let train the educative community in real time actions in solving problems between parents and sons interactions according to the affective - academic habits that are generated in the institutional and home-like students' environment.

Key words: Pedagogical strategy, parent, academic performance. 


\section{Introducción}

Alcanzar la calidad de la educación es uno de los retos que actualmente vienen trabajando las diferentes instituciones educativas en Colombia; por ende hablar del rendimiento académico de los estudiantes de educación básica primaria se convierte en un tema fundamental de discusión en la medida en que se buscan alternativas de intervención que permitan contrarrestar dicha situación, dadas las reiteradas calificaciones bajas que se tienen en los en los informes que se generan al término de cada periodo escolar.

En consecuencia, se supone que los entes formadores del estudiante: sociedad, escuela y familia, han aunado esfuerzos para dar cumplimiento a este precepto. Lo que actualmente se evidencia es que la familia ha ido transfiriendo a la escuela su responsabilidad en la formación de sus hijos, dejando en manos de la misma los procesos de aprendizaje. Situación que da lugar a que la escuela actualmente presente dificultades para alcanzar su misión social, al darse a no sincronizarse las estrategias pedagógicas para hacer que el trabajo escolar tenga continuidad en el hogar $y$, de esta manera, alcanzar un rendimiento académico y social satisfactorio del estudiante.

En este contexto, la Institución Educativa María Cristina Arango Sede Los Pinos, viene presentando durante los últimos años bajo rendimiento académico, puesto que son varios los estudiantes que en el nivel de educación básica primaria, al finalizar el año escolar, evidencian un número significativo de asignaturas no aprobadas, situación que no les permite alcanzar satisfactoriamente sus metas académicas. Ante este panorama es fundamental señalar que los problemas relacionados con el bajo rendimiento académico deben ser tomados en cuenta con mayor objetividad y para ello es pertinente considerar a la familia como la "unidad central encargada de la integración social primaria del niño"(Pacheco, 2000, p. 89).

A partir de lo anterior, se define como objetivo general determinar el grado de influencia de la Escuela de Padres como estrategia pedagógica para mejorar el rendimiento académico de los estudiantes de educación básica primaria de la Sede Los Pinos de la Institución Educativa María Cristina Arango. 
Este análisis permite entregar a la comunidad educativa de la Institución un estudio serio y riguroso sobre la influencia de la escuela de padres en el rendimiento académico de los estudiantes, toda vez que da lugar a caracterizar las diferentes maneras de acompañamiento de los padres de familia en los procesos académicos de sus hijos, y analizar comparativamente los resultados académicos de los estudiantes, hijos de padres que asisten al programa de escuela de padres y de quienes no asisten.

\section{Revisión de la literatura}

El presente artículo fundamenta su posición con base en diferentes referentes teóricos e investigativos, que exponen elementos relacionados con la educación primaria, estrategias pedagógicas, rendimiento académico y núcleo familiar, así como información relevante sobre los referentes normativos que rigen el programa de escuela de padres.

Desde esta perspectiva, se debe iniciar este apartado señalando que el concepto de rendimiento académico se encuentra interrelacionado con el concepto de inteligencia, en la medida en que las variables cognitivas tienen estrecha relación con las experiencias que el estudiante realiza en su vida escolar. Por tal motivo, haciendo referencia en lo que respecta a una inteligencia escolar, se retoma lo expuesto por Bravo (1990), quien expone la siguiente definición sobre inteligencia escolar:

Es el conjunto de habilidades cognitivas y verbales que procesan, integran y organizan el aprendizaje y toda la experiencia escolar y lo van relacionando con los aprendizajes y experiencias anteriores, por medio de la codificación y categorización de sus contenidos, de modo de permitir la aplicación a situaciones nuevas. (p.68).

Lo anterior permite conocer que la inteligencia escolar es aquella capacidad que tiene el estudiante de asimilar la experiencia y la enseñanza de la vida escolar para luego llevarlas a la práctica en diversas situaciones que experimenta a diario, ya sea dentro o fuera de su entorno escolar.

De igual manera, Pizarro (1985) incluye una definición de Rendimiento Académico que permite conocer con mayor apropiación y claridad este 
concepto, al definirlo como "una medida de las capacidades respondientes o indicativas que manifiestan, en forma estimativa, lo que una persona ha aprendido como consecuencia de un proceso de instrucción o formación" (p. 16). Esta definición evidencia que el rendimiento académico es un resultado, interpretado en competencias o logros educativos, de las capacidades y habilidades del estudiante frente a un conjunto de estímulos educativos.

En este sentido, son diversos los estudios que se han desarrollado para definir los factores que inciden de manera significativa en el bajo rendimiento académico de los estudiantes, en especial los de educación primaria. Por ende, se trae a referencia a Jiménez (2008), quien presenta de forma muy objetiva los diversos factores que inciden ampliamente en el rendimiento académico, señalando:

"Los padres son piezas fundamentales para que sus hijos alcancen un aprovechamiento deseable en las aulas. Los conflictos familiares, el trabajo y la falta de motivación y preparación que existe entre los padres, impiden obtener resultados satisfactorios en los estudiantes. La orientación profesional dirigida a los padres es una necesidad para combatir el bajo rendimiento en los niños" (Pág. 1).

En consecuencia, se puede señalar que la familia, es sin duda, un factor determinante en el bajo rendimiento académico de los estudiantes, en la medida en que este núcleo es la primera escuela del niño mediante la cual se adquieren las primeras nociones de la vida, se forman los valores y se fundamentan las capacidades para actuar frente a un mundo social. Por ende, es fundamental indicar que "en la familia, los padres se consideran guías, cuya tarea es la de transmitir a sus hijos normas de comportamiento verdaderamente humanas y reconocer en cualquier situación sus aciertos y sus desaciertos" (Vallejo, 1989, p. 2).

Es evidente la situación actual de las familias en Colombia, cuando se a diario se presentan diversas situaciones de agresividad entre las mismas, como consecuencia de la carencia de tiempo, de afecto, de tolerancia, autoestima y la notable desintegración. La formación de madres y padres es todavía algo casi anecdótico en la mayoría de los centros escolares, por lo que es consecuente considerar, como lo hacen Beltrán y Pérez (2002) que "la familia no es solo el medio afectivo, tranquilizador y estimulante 
que favorece un pleno desarrollo, sino también es el medio para la adaptación y el desarrollo social". Continúan estos autores afirmando que el niño "descubre en la familia el contacto con el otro y con el grupo, la coacción, las prohibiciones, las limitaciones" (p.3). Por eso, cuando los padres no tienen unos criterios educativos claros, consecuentes y adoptan actitudes educativas opuestas entre ellos, esto da origen a que los hijos sean más inseguros y desorganizados en el estudio y sus esfuerzos escolares suelen ser bastantes inconsistentes.

A este panorama la Institución Educativa María Cristina Arango no ha sido ajena, por lo que se hace evidente señalar el compromiso de los padres de familia y de la comunidad educativa para brindar mejores posibilidades que permitan al estudiante tener la capacidad de enfrentar los retos académicos que a diario le son presentados. Es aquí donde encuentra justificación la acción docente y la capacitación de padres de familia, para que desde la escuela y la casa se contribuya a la cualificación de la familia y a la necesidad de su compromiso para el logro del óptimo rendimiento académico.

Conviene destacar que la escuela de padres, como alternativa pedagógica, ofrece la oportunidad de integrar a padres en las actividades educativas, fomentando acciones colectivas con el fin de transformar progresivamente a la institución escolar en una verdadera comunidad de ciudadanos con metas comunes, orientados hacia el perfeccionamiento de la formación ética e intelectual de los alumnos, y que como miembros de la comunidad escolar deben incorporarse al proceso de aprendizaje organizacional institucional. En otras palabras, la Escuela de Padres representa un espacio de aprendizaje, de reflexión colectiva e intercambios de experiencias entre los padres y estudiantes del centro educativo, con lo que se busca alcanzar la capacidad de mejorar la gestión educativa en el centro correspondiente (Ramírez, 2012, p. 12).

En Colombia la escuela de padres se enmarca en su legalidad de acuerdo con la Ley 1404 de 2010, mediante la cual se crea el programa Escuela para Padres en las instituciones de Educación Preescolar, Básica y Media del país. Dicha normatividad señala en el artículo primero que el propósito fundamental de dicho programa es integrar a todos los padres y madres de familia, así como a los acudientes, a un cuerpo organizado que se articule 
con la comunidad educativa, principalmente docentes, alumnos y directivos, asesorados por profesionales especializados, para pensar en común, intercambiar experiencias y buscar alternativas de solución a la problemática que se presente en la formación de los hijos e hijas, la recuperación de valores, el fortalecimiento de instrumentos adecuados en técnicas de estudio y la comunicación e integración de la familia.

\section{Métodos y materiales}

Se llevó a cabo una investigación de estudio de casos (carácter comparativo) mediante un enfoque cualitativo, entendiendo el estudio de caso como un método que se ha utilizado ampliamente para comprender a profundidad la realidad social y educativa; igualmente, analiza diversos fenómenos contemporáneos, que representan algún tipo de problemática de la vida cotidiana (Barrio, et al., 2005). Por ende, se conformaron dos grupos de trabajo con estudiantes de educación primaria de la Institución Educativa María Cristina Arango, Sede Los Pinos, los cuales se clasificaron en grupo A (estudiantes con bajo rendimiento académico y sus respectivos padres), y grupo $B$ (estudiantes que no presentan bajo rendimiento académico y sus respectivos padres).

A partir de lo anterior, el estudio de la problemática implicó un proceso de comparación en donde se intentó presentar la realidad de la misma desde las propias acciones de los actores (experiencias, actitudes, pensamientos, reflexiones), ya que se interactuó con cada uno de ellos (docentes, estudiantes y directivos), mediante un acercamiento y comunicación permanentes, de tal forma que se pudiera determinar el grado de influencia de la Escuela de Padres en el mejoramiento del rendimiento académico de los estudiantes. La información obtenida se convirtió en un insumo fundamental para la construcción de los análisis respectivos.

Fundamental señalar que en el marco de esta investigación se asume el proceso de descripción como:

Una estrategia de investigación dirigida a comprender las dinámicas presentes en contextos singulares, la cual podría tratarse del estudio de un único caso o de varios casos, combinando distintos métodos para la recogida de evidencia cualitativa y/o cuantitativa con el fin de describir, verificar o generar teoría (Martínez, 2010). 
Desde esta perspectiva se llevó a cabo una caracterización de las diferentes maneras de acompañamiento de los padres de familia en los procesos académicos de sus hijos, y posteriormente se realizó un análisis comparativo de los resultados académicos de los estudiantes, hijos de padres que asisten al programa de Escuela de Padres y de quienes no asisten.

En lo concerniente a la población, la investigación se desarrolló en la Institución Educativa María Cristina Arango, Sede Los Pinos, con la comunidad académica que la integra, la cual fue objeto de estudio y análisis. De igual forma, se utilizaron unos instrumentos que garantizaron la obtención de la información, relacionada con la influencia de la escuela de padres como estrategia pedagógica para mejorar el rendimiento académico de los estudiantes. Estos instrumentos fueron:

Análisis documental: Los documentos que se tuvieron en cuenta para revisión y análisis fueron el observador del alumno, a través del cual es posible obtener información valiosa para determinar los factores que influyen en el rendimiento académico de los estudiantes, y el boletín académico de los mismos, realizándose una comparación de los resultados académicos de los estudiantes hijos de padres que asisten al programa de Escuela de Padres y de quienes no asisten, para analizar el grado de influencia de la implementación de esta estrategia pedagógica.

Entrevista estructurada: se realizaron entrevistas de manera personalizada a los docentes, a los padres de familia y a los estudiantes, las cuales fueron entendidas como una conversación oral, con el objetivo de reconocer la Escuela de Padres como estrategia pedagógica de acompañamiento a los procesos de aprendizaje escolar. Se utilizó un cuestionario guía y se hizo uso de la grabación magnetofónica.

\section{Resultados}

La aplicación de los diferentes instrumentos de investigación permitió obtener los siguientes resultados del Grupo A:

Se observó de manera significativa que el rendimiento académico de estos estudiantes se ve afectado por la falta de compromiso de los mismos y de 
los padres de familia, en relación con la utilización del tiempo en la jornada contraria a la escolar, dado que un porcentaje mayor de estudiantes dedican gran parte de su tiempo para ver televisión; en forma paralela, otros estudiantes dedican su tiempo a las actividades recreativas sin ningún control, y un menor número de estudiantes se ve afectado en su rendimiento académico por no asistir en forma regular a clases.

De igual forma, un porcentaje alto de los padres de familia señalaron que no les pueden brindar el acompañamiento escolar que requieren sus hijos, en cuanto al desarrollo de tareas y actividades escolares, debido a que sus obligaciones laborales no se lo permiten, y un porcentaje similar de padres de familia argumentaron que no lo hacen debido a que su nivel de escolaridad es muy bajo y, por consiguiente, no tienen las competencias para guiar a sus hijos en los temas académicos. No obstante, la totalidad de los padres de familia en las entrevistas estuvieron de acuerdo en que la familia es decisiva e importante para que el estudiante pueda obtener buenos resultados académicos.

De la misma manera, la mayoría de los estudiantes entrevistados consideran que tuvieron bajo rendimiento académico por la falta de apoyo en casa para desarrollar sus compromisos académicos. Otro número significativo de estudiantes argumenta que hay unas tareas que ellos no realizan o que están mal elaboradas, porque no cuenta con un apoyo o asesoría frente a la temática de la misma y por consiguiente obtienen malas notas.

En relación con el programa de Escuela de Padres, los entrevistados reconocen que esta estrategia es un medio de asesoría que les permite analizar problemáticas propias de sus hijos y, a la vez, buscar actividades que les permitan superar dificultades. Un número menor de entrevistados señalaron que en alguna ocasión habían oído mencionar sobre el programa, pero no conocen su finalidad.

Al indagar en los estudiantes sobre el interés de contar con acompañamiento profesional psicopedagógico u orientación escolar que permitan ofrecer una ayuda para superar las dificultades académicas, estos respondieron en su totalidad de manera afirmativa argumentando que requieren de un trabajo mancomunado con sus padres y docentes para superar las dificultades académicas. 
Por otra parte, la aplicación de los diferentes instrumentos de investigación permitió obtener los siguientes resultados del Grupo B:

Gran parte de los padres de familia encuestados consideran que el problema del bajo rendimiento académico de los estudiantes radica en la falta de acompañamiento de los acudientes en las diferentes actividades académicas de los niños; por lo tanto, argumentan que es fundamental asistir a sus hijos en el desarrollo de los compromisos académicos. Además afirman que han recurrido a la ayuda de alguna otra persona para la resolución de algunas de estas actividades.

Por otra parte, todos los padres de familia encuestados respondieron que el hogar es un pilar fundamental en el buen desempeño académico de los estudiantes, debido a que esto les ayuda a superar las dificultades y a mejorar la confianza en sí mismos. Por lo que asisten participativamente en el programa escuela de Padres que ofrece la institución educativa, ya que reciben orientación pedagógica y profesional para atender las necesidades académicas de sus hijos y ayudarlos durante su proceso de formación.

De la misma manera, la mayoría de los estudiantes entrevistados consideran que su buen rendimiento académico se ha dado en la medida en que son conscientes de su papel en la escuela y su grado de responsabilidad en las tareas y actividades académicas. Además señalan que sus padres de familia están atentos a sus deberes escolares y ofrecen una buena asesoría en cuanto afirman que estos tienen conocimientos suficientes para poder ayudarles en la solución de sus tareas.

En cuanto a la opinión de los docentes y directivos de la institución educativa, estos señalan que la desintegración familiar, los problemas económicos de las familias, la falta de escolarización de los padres, el sistema de evaluación relacionado con la promoción automática, son factores que inciden significativamente en el rendimiento académico de los estudiantes. De igual forma, argumentan la influencia positiva que ha tenido el programa Escuela de Padres, pues permite atender las dificultades académicas de los estudiantes con su núcleo familiar, además que los compromete en el proceso de formación de sus hijos. 


\section{Discusión}

Los resultados arrojados en el desarrollo de la investigación permiten señalar que los estudiantes de la educación básica primaria de la Institución Educativa María Cristina Arango de Pastrana pertenecientes a la Sede Los Pinos presentan un bajo rendimiento académico como consecuencia de la ausencia de acompañamiento de los padres de familia en su proceso de formación escolar.

Lo anterior tiene lugar al encontrar que los estudiantes del grupo de estudio A dedican varias horas de su tiempo de ocio a ver televisión y jugar; en cambio dedican un tiempo muy mínimo para sus compromisos escolares, lo que permite evidenciar la falta de acompañamiento de los padres de familia en el fomento de la responsabilidad frente a las deberes académicos. Es fundamental señalar que una de las manifestaciones del bajo rendimiento académico de estos estudiantes es la falta de disciplina en los hogares, donde no tienen un horario establecido para las actividades escolares y recreativas.

Situación contraria se pudo evidenciar en los estudiantes del grupo de estudio $B$, quienes tienen como prioridad atender sus obligaciones escolares, lo que ha permitido obtener un mejor desempeño. Lo anterior ha tenido lugar dado a que sus padres han fomentado responsabilidad y les han enseñado a hacer uso de diferentes medios de estudio para desarrollar sus compromisos académicos.

Por consiguiente, es preciso señalar, teniendo en cuenta el principio de corresponsabilidad que consignar tanto la Constitución Política Nacional como el Código de Infancia, que el proceso educativo requiere del compromiso constante de los padres de familia, estudiantes y docentes y, en forma paralela, de las entidades oficiales encargadas de realizar el seguimiento al sistema educativo, para garantizar la calidad de la educación y por ende la promoción académica se los educandos.

Se hace indispensable y urgente desarrollar al interior de las instituciones educativas proyectos de acompañamiento a los padres de familia como lo es el programa Escuela de Padres, cuya estrategia pedagógica permite brindar capacitaciones oportunas que permitan superar problemáticas 
propias de la inter-acción padres e hijos, en lo relacionado con la parte afectiva-académica, hábitos de estudios y los que se generen propios de la dinámica del contexto institucional y de los hogares de los estudiantes. Finalmente se señala que la formación de los docentes y la asignación en sus áreas de especialización en forma paralela a los contenidos que desarrollan son fundamentales en el éxito académico.

\section{Conclusiones}

De acuerdo con los resultados obtenidos en el desarrollo del presente artículo, es evidente que el rendimiento académico es un indicador de la eficacia y la eficiencia de los procesos académicos de la institución y del trabajo mancomunado de toda la comunidad educativa; es decir, que no solo el estudiante tiene responsabilidad en el logro de sus metas escolares, sino también, los docentes y los padres de familia, entre otros. Por tal motivo, y aunque es notorio que existe un bajo rendimiento académico de algunos de los estudiantes de la Institución Educativa María Cristina Arango de Pastrana, Sede los Pinos, es posible generar cambios en el desempeño académico de los mismos, debido a que sus causas han sido claramente identificadas y reconocidas como falencias. Por ende se concluye que la problemática central del bajo rendimiento académico, está directamente relacionado con: la falta de acompañamiento por parte de los padres de familia, la formación de hábitos que conlleven a la disciplina escolar, y la carencia de pautas o normas que establezcan horarios y permitan cumplir con los compromisos escolares, sin dejar de contar con un tiempo para la sana recreación.

Por lo anterior se propone el programa Escuelas de Padres como alternativa pedagógica para ofrecer a los padres de familia de la Institución Educativa María Cristina Arango de Pastrana, Sede los Pinos, información y conocimientos necesarios sobre diferentes temas, con el fin de brindar una mayor capacitación para ejercer su función; de igual manera, este programa facilita recursos educativos y formativos para promover en sus hijos actitudes, valores, habilidades personales y sociales sanas que les permitan afrontar, de manera responsable, la realidad de sus vidas.

Por medio de este programa se promueven y fortalecen las relaciones entre escuela-comunidad y entre comunidad-escuela con lo cual se favorece 
el mejoramiento de la conducta ciudadana y comunitaria de los niños y adolescentes. Además, se da lugar a la formación de autoestima entre los integrantes de la comunidad educativa, mediante la capacitaciones que se brindan, dando lugar a que la institución se convierta en un eje integrador de las acciones que habrán de impulsar el desarrollo comunitario.

Finalmente, el propósito de la Escuela de Padres es invitar a los padres de familia hacia el conocimiento y la valoración de la labor que los docentes realizan con sus hijos y de ese modo, promover una actitud de solidaridad y respeto entre los mismos, lo que genera un clima mancomunado de trabajo que propicia un excelente rendimiento académico de los estudiantes. La Escuela de Padres constituye una herramienta que tiene una importancia de primera magnitud para la dirección del centro educativo, la cual se pone de manifiesto a través de los beneficios que habrá de reportar en apoyo a la misión de dirigir, por cuanto ofrece una ayuda y le da facilidad en la labor de organizar la escuela.

\section{Referencias Bibliográficas}

- Barrio, I., González, J., Padín, L., Peral, P., Sánchez, I., Tarín, E. (2005). El estudio de caso: método de investigación educativa. Universidad Autónoma de Madrid. Recuperado de: https://www.uam.es/ personal_pdi/stmaria/jmurillo/investigacion EE/

- Beltrán, J. \& Pérez, L. (2002). Educar para el siglo XXI. Pensar, crecer y convivir en familia. Madrid, Editorial CCS.

- Bravo, L. (1990). Psicología de las dificultades del aprendizaje escolar. Santiago de Chile: Editorial Universitaria

- Congreso de Colombia. Ley 1404 de 2010. Por la cual se crea el programa escuela para padres y madres en las instituciones de educación preescolar, básica y media del país. Bogotá, Julio 27.

hijos..html\#.VaUS8F9_Oko.

- Jiménez Villacís, M. (2008). Influencia de los padres en el rendimiento escolar de sus hijos. Universidad Anáhuac Mayab. Recuperado en: http:/ 
Revista Paca No. 8

/codice.anahuacmayab.mx/2117-1-

influencia+de+los+padres+en+el+rendimiento+escolar+de+sus+

- Pizarro, Ana, Rasgos y actitudes del profesor efectivo, Tesis de Magister en Ciencias de la Educación, Pontificia Universidad Católica de Chile, Santiago, 1985, p, 16 Presentaciones/Est_Casos_doc.pdf

- Ramírez, A. (2012). Importancia de "La escuela de padres y madres". -Recuperado de: http://laescuelaparapadress.blogspot.com/

- Vallejo, J. (1989). Relaciones Humanas. Editores e Impresores Bristol, Décima edición. Bogotá. 